\title{
CRITICAL EXPERIMENT OF THORIUM LOADED THERMAL CORES AT KUCA (1) A NEW CRITICAL EXPERIMENT OF THORIUM LOADED CORE WITH HARDER NEUTRON SPECTRUM IN KUCA
}

\author{
Tadafumi Sano $^{1^{*}}$, Jun-ichi Hori², Jeaong Lee², Yoshiyuki Takahashi², \\ Kazuki Takahashi ${ }^{3}$ and Hironobu Unesaki ${ }^{2}$ \\ ${ }^{1}$ Kindai University Atomic Energy Research Institute \\ 3-4-1, Kowakae, Higashiosaka-shi, Osaka, Japan \\ ${ }^{2}$ Institute for Integrated Radiation and Nuclear Science, Kyoto University \\ 2-1010, Asashiro-nishi, Kumatori-cho, Sennan-gun, Osaka, Japan \\ ${ }^{3}$ Graduate School of Science and Engineering, Kindai University \\ 3-4-1, Kowakae, Higashiosaka-shi, Osaka, Japan
}

t-sano@kindai.ac.jp, hori@rri.kyoto-u.ac.jp,zelloseve@naver.com, ytaka@rri.kyoto-u.ac.jp, takahashi.kazuki0524@gmail.com,unesaki@rri.kyoto-u.ac.jp

\begin{abstract}
In order to perform integral evaluation of ${ }^{232} \mathrm{Th}$ capture cross section, a series of critical experiments for thorium-loaded and solid-moderated cores in KUCA had been carried out. In these experimental cores, $\mathrm{H} /{ }^{235} \mathrm{U}$ nuclide ratio ranged about from 150 to 315 , and ${ }^{232} \mathrm{Th} /{ }^{235} \mathrm{U}$ nuclide ratio ranged about from 13 to 19 . In this study, a new critical experiment with Th loaded core in KUCA, which had about 70 of the $\mathrm{H} /{ }^{235} \mathrm{U}$ ratio and 12.7 of ${ }^{232} \mathrm{Th} /{ }^{235} \mathrm{U}$ ratio, was carried out. As results, the excess reactivity was $0.086 \pm 0.003(\% \mathrm{dk} / \mathrm{k})$ and the $\mathrm{k}_{\text {eff }}$ was $1.0009 \pm 0.0003$, where the effective delayed neutron fraction was $7.656 \mathrm{E}-3$. The $\mathrm{k}_{\text {eff }}$ was also calculated by MVP3.0 with different nuclear libraries. The respective calculations with JENDL-4.0, JENDL-3.3 and ENDF/B-VII.0 lead to $1.0056 \pm 0.0086(\%), 1.0048 \pm 0.0085$ (\%) and $1.0056 \pm 0.0086(\%)$.On the other hand, the further MVP3.0 calculations, where only the ${ }^{232} \mathrm{Th}$ cross sections were taken from JENDL-4.0, JENDL-3.3 or ENDF/B-VII.0 but all other nuclides were done from JENDL-4.0, were carried out to examine an impact of the difference of ${ }^{232} \mathrm{Th}$ cross section among these nuclear libraries to the $\mathrm{k}_{\text {eff. }}$ The $\mathrm{k}_{\text {eff }}$ calculated with respective ${ }^{232} \mathrm{Th}$ cross sections from JENDL-3.3 and ENDF/B-VII.0 was 1.0038 $\pm 0.0086(\%)$ and $1.0040 \pm 0.0086(\%)$.
\end{abstract}

KEYWORDS: KUCA, ${ }^{232} \mathrm{Th}$, Critical Experiment, Crosse Section, Nuclear Data Library, Sensitivity

\section{INTRODUCTION}

In discussing the feasibility of a thorium-based reactor system from an engineering perspective, it is necessary to verify and evaluate the impact of thorium (Th) utilization on the accuracy and reliability of core nuclear characteristic parameters such as criticality, conversion rate, and fuel balance. Improving the accuracy of the nuclear characteristics of Th is an important R \& D step in the Th utilization reactor 
system. However, compared to the currently uranium / plutonium-based light water reactor system, the Th-based nuclear reactor system has not been sufficiently researched on the following two viewpoints. One is to evaluate the reliability and uncertainty of cross section of related nuclides including ${ }^{232} \mathrm{Th}$ in the nuclear data library. The other is the effect of the cross section uncertainties on the nuclear characteristics in the Th-based reactor core.

The authors have systematically carried out critical experiments on Th loaded cores using Kyoto University Critical Assembly (KUCA). In KUCA, the 9 critical experiments have been carried out with 3 type $\mathrm{H} /{ }^{235} \mathrm{U}$ nuclide ratios (about $150,210,315$ ) and 3 type ${ }^{232} \mathrm{Th} /{ }^{235} \mathrm{U}$ nuclide ratios in a Th loaded fuel shown in Table I [1]. Integral evaluations of thorium loaded thermal neutron systems and ${ }^{232} \mathrm{Th}$ capture cross sections has been performed via those critical experiments.

In this study, from the viewpoint of expanding critical experimental data, an experiment was conducted by a Th fuel loaded core with 70 of $\mathrm{H} / 235 \mathrm{U}$ and 12.7 of ${ }^{232} \mathrm{Th} /{ }^{235} \mathrm{U}$ ratio at KUCA. Section 2 outlines the critical experiment at KUCA, Section 3 describes the numerical analysis and sensitivity analysis for the criticality, and Section 4 concludes.

Table I. List of experiments conducted by KUCA with thorium fuel loaded core.

\begin{tabular}{|c|c|c|c|}
\hline $\mathrm{H}^{235} \mathrm{U}$ & 12.7 & 15.2 & 19 \\
\hline 70 & This study & --- & --- \\
\hline 150 & 0 & $\mathrm{O}$ & 0 \\
\hline 210 & O & O & 0 \\
\hline 315 & 0 & 0 & 0 \\
\hline
\end{tabular}

\section{CRITICAL EXPERIMENT AT KUCA}

\subsection{Core Configuration with Thorium loaded fuel at KUCA}

KUCA solid moderator core is able to consist of enriched uranium fuel plate and various moderator plates (i.e. polyethylene and graphite). Figure 1 shows the schematic view of KUCA solid moderator core. In order to vary the neutron spectrum of the irradiation field, the $\mathrm{H} /{ }^{235} \mathrm{U}$ nuclide ratio in the unit cell is varied by the combination of the U-Al alloy fuel plates (EU) and the moderator plates. The enrichment of the uranium is about $93 \mathrm{wt} \%$. The width of the plates have 2 inch square. The thickness of the fuel plates has $1 / 16$ inch. In this study, a new critical core consisted of two type fuel elements. One was a Th loaded fuel element (referred to as "Th fuel"), and the other was a driver fuel element ("Driver fuel"). Figure 1 shows the configuration of Th fuel and the Driver fuel. The Th fuel consisted of 27 unit cells and sandwiched by a upper and a lower polyethylene reflector. The unit cell has three EU plates, one Th plate of $1 / 8$ " thickness and two polyethylene plates of $1 / 8$ " thickness. The Driver fuel has 49 unit cells and the unit cell was composed of one EU plate and two polyethylene plates. The experimental core was consisted of 37 Th fuels and 17 Driver fuels shown in Figure 2. Where "C" means a control rod and "S" means a safety rod. In addition, the partial driver fuel element which has 17 or 19 unit cells were loaded to adjust an excess reactivity of the critical core. The Th fuels were loaded at the center of the core and surrounded by the Driver fuels and the partial driver fuels. 


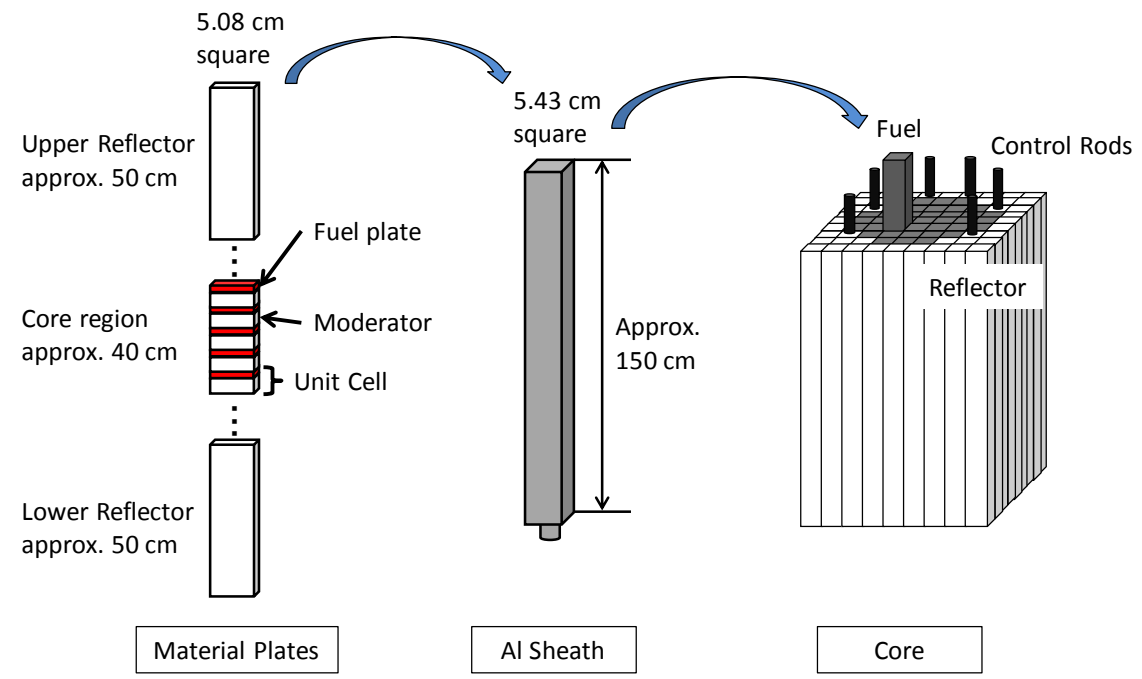

Figure 1. Schematic view of KUCA solid moderator core.

Th fuel (F) 1/16"EU+1/8"Th+1/16"EU+1/16"EU+1/8"PE+1/8"PE : Unit cell

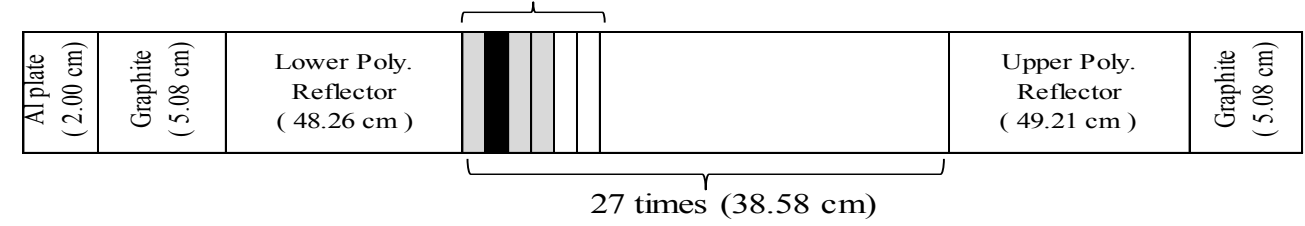

Driver fuel(D) 1/16"EU+1/8"PE+1/8"PE : Unit Cell

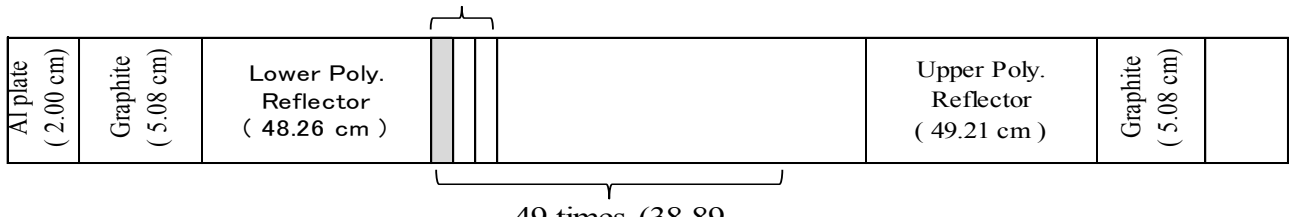

49 times $(38.89$

Figure 2. Configuration of Th fuel and Driver fuel

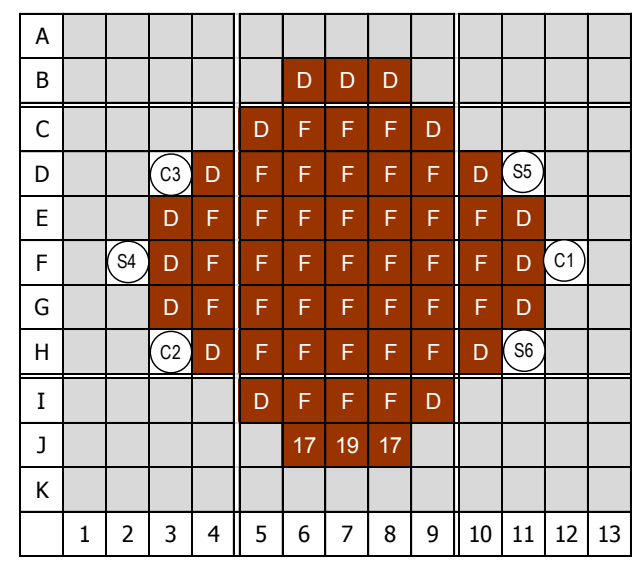

Figure 3. Core Configuration. 


\subsection{Results of Critical Experiments}

In order to obtain an effective multiplication factor of the core described in the previous section, excess reactivity measurement was performed 6 times by the reactor period method, respectively. In each measurement, the $\mathrm{C} 1$ and $\mathrm{C} 2$ control rods were set to the upper limit and the $\mathrm{C} 3$ control rod was adjusted to the critical position. And the $\mathrm{C} 3$ control rod was drawn to the upper limit from the critical position and the reactivity was inserted into the core. The results of measured excess relativities and the control rod position are shown in Table II. Here, the kinetics parameters using the reactivity measurements were calculated by the continuous energy Monte-Carlo code MVP3.0 [2] with the JENDL-4.0 [3]. The parameters are shown in Table III. As the result, the excess reactivity was $0.0865 \pm 0.0026(\% \mathrm{dk} / \mathrm{k})$ and the $\mathrm{k}_{\text {eff }}$ was $1.0009 \pm 0.0003$.

Table II. Measured excess reactivity.

\begin{tabular}{cccccc}
\hline Run No. & C1 $(\mathrm{mm})$ & $\mathrm{C} 2(\mathrm{~mm})$ & $\mathrm{C} 3(\mathrm{~mm})$ & $\begin{array}{c}\text { Period } \\
(\mathrm{sec})\end{array}$ & $\begin{array}{c}\text { Excess reactivity } \\
(\% \mathrm{dk} / \mathrm{k})\end{array}$ \\
\hline $9577-01$ & 1201.15 & 1206.11 & 735.63 & 82.77 & 0.0876 \\
9581 & 1201.16 & 1201.11 & 736.99 & 84.19 & 0.0864 \\
9582 & 1201.15 & 1201.11 & 736.23 & 83.78 & 0.0868 \\
9584 & 1201.11 & 1201.13 & 739.61 & 90.80 & 0.0816 \\
9586 & 1201.15 & 1201.11 & 734.78 & 82.95 & 0.0874 \\
9588 & 1201.18 & 1201.12 & 732.62 & 80.59 & 0.0893 \\
\hline
\end{tabular}

Table III. Kinetic parameter.

\begin{tabular}{cc}
\hline$\beta$ eff & $\wedge$ \\
\hline $7.658 \mathrm{E}-03$ & $3.260 \mathrm{E}-05$ \\
\hline
\end{tabular}

\section{NUMERICAL ANALSYS}

\subsection{Criticality with various nuclear data libraries}

Numerical analysis for the criticality ( $\mathrm{k}_{\mathrm{eff}}$ ) in the critical experimental core was performed using MVP3.0, JENDL-4.0, JENDL-3.3 [4], and ENDF / B-VII.0 [5]. The Monte Carlo runs performed for 5,000 active and 100 inactive batches with 20,000 histories per batch. The numerical results of the $\mathrm{k}_{\mathrm{eff}}$ are shown in Table IV. The respective calculations with JENDL-4.0, JENDL-3.3 and ENDF/B-VII.0 lead to 1.0056 $\pm 0.0086(\%), 1.0048 \pm 0.0085(\%)$ and $1.0056 \pm 0.0086(\%)$. The calculate results have bias of about $0.39(\% \mathrm{dk} / \mathrm{k})$ to $0.47(\% \mathrm{dk} / \mathrm{k})$ compared to the experimental result. This bias are consistent with the analysis of previous experimental results for criticality [6]. On the other hand, the differences of calculated $\mathrm{k}_{\mathrm{eff}}$ between JENDL-4.0 and other libraries are less than $0.08(\% \mathrm{dk} / \mathrm{k})$. The average neutron spectrum in the Th loaded region is shown in Figure 4. The present core has harder neutron spectrum than the previous cores.

Table IV. Results of numerical analysis for the criticality.

\begin{tabular}{cccc}
\hline Experiment & JENDL-4.0 & JENDL-3.3 & ENDF/B-VII.0 \\
\hline 1.0009 & 1.0056 & 1.0048 & 1.0056 \\
$\mathrm{C} / \mathrm{E}$ & 1.0047 & 1.0039 & 1.0047 \\
\hline
\end{tabular}




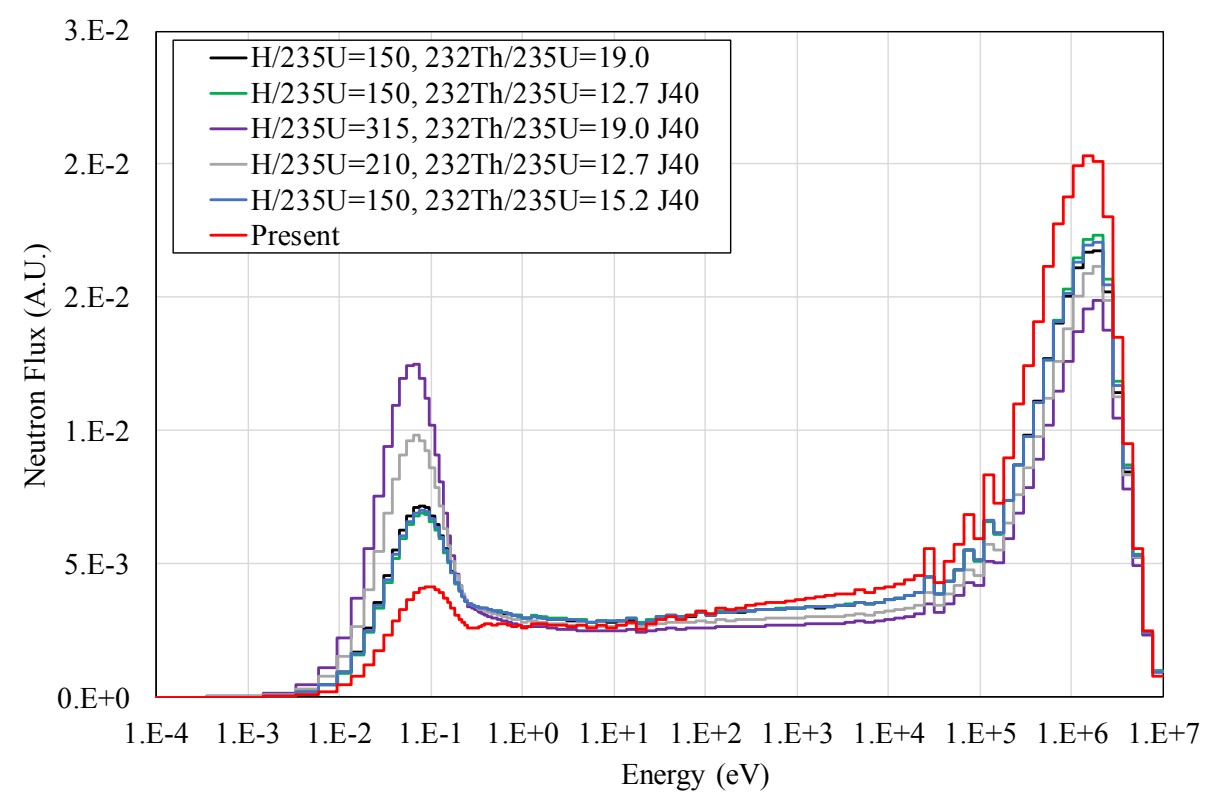

Figure 4. Neutron spectrum in each core.

\subsection{Impact of ${ }^{232} \mathrm{Th}$ cross section for criticality calculation}

The further MVP3.0 calculations, where only the ${ }^{232} \mathrm{Th}$ cross sections were taken from JENDL-4.0, JENDL-3.3 or ENDF/B-VII.0 but all other nuclides were done from JENDL-4.0, were carried out to examine an impact of the difference of ${ }^{232} \mathrm{Th}$ cross section among these nuclear libraries to the $\mathrm{k}_{\text {eff. }}$ The results are shown in Table $\mathbf{V}$. The $\mathrm{k}_{\text {eff }}$ calculated with respective ${ }^{232} \mathrm{Th}$ cross sections from JENDL-3.3 and ENDF/B-VII.0 are $1.0038 \pm 0.0086(\%)$ and $1.0040 \pm 0.0086(\%)$, respectively. The differences of the $\mathrm{k}_{\text {eff }}$ due to ${ }^{232} \mathrm{Th}$ cross section change from JENDL-4.0 are obtained to be 0.180 (\%dk/k) of JENDL3.3 and $0.162(\% \mathrm{dk} / \mathrm{k})$ of ENDF/B-VII.0

Table V. Effect of ${ }^{232} \mathrm{Th}$ cross section change on $k_{\text {eff }}$ calculation.

\begin{tabular}{lccc}
\hline Case & $\mathrm{k}$-eff & difference $(\% \mathrm{dk} / \mathrm{k})$ & $\mathrm{C} / \mathrm{E}$ \\
\hline JENDL-4.0 & 1.0056 & ---- & 1.0047 \\
JENDL-4.0 $\rightarrow$ JENDL-3.3 & 1.0038 & -0.178 & 1.0029 \\
JENDL-4.0 $\rightarrow$ ENDF/B-VII.0 & 1.0040 & -0.158 & 1.0031 \\
\hline
\end{tabular}

The main contribution of $\mathrm{k}_{\text {eff }}$ difference is ${ }^{232} \mathrm{Th}$ capture cross section. To discuss the changes of $\mathrm{k}_{\mathrm{eff}}$, the differences of ${ }^{232} \mathrm{Th}$ capture cross section between JENDL-4.0 and other libraries are shown in Figure 5. Comparison between JENDL-4.0 and JENDL-3.3, the capture cross section in JENDL-3.3 is larger than JENDL-4.0 from energy range of $0.1(\mathrm{eV})$ to $40(\mathrm{eV})$, and it is smaller than JENDL-4.0 in the energy region higher than $40(\mathrm{eV})$. Here, the major difference of the capture cross section is "resonance valley" with a small cross section. The difference of capture cross section between JENDL-4.0 and ENDF / BVII.0 is small. In addition, the energy region below $3.3(\mathrm{keV})$ has the same value. 


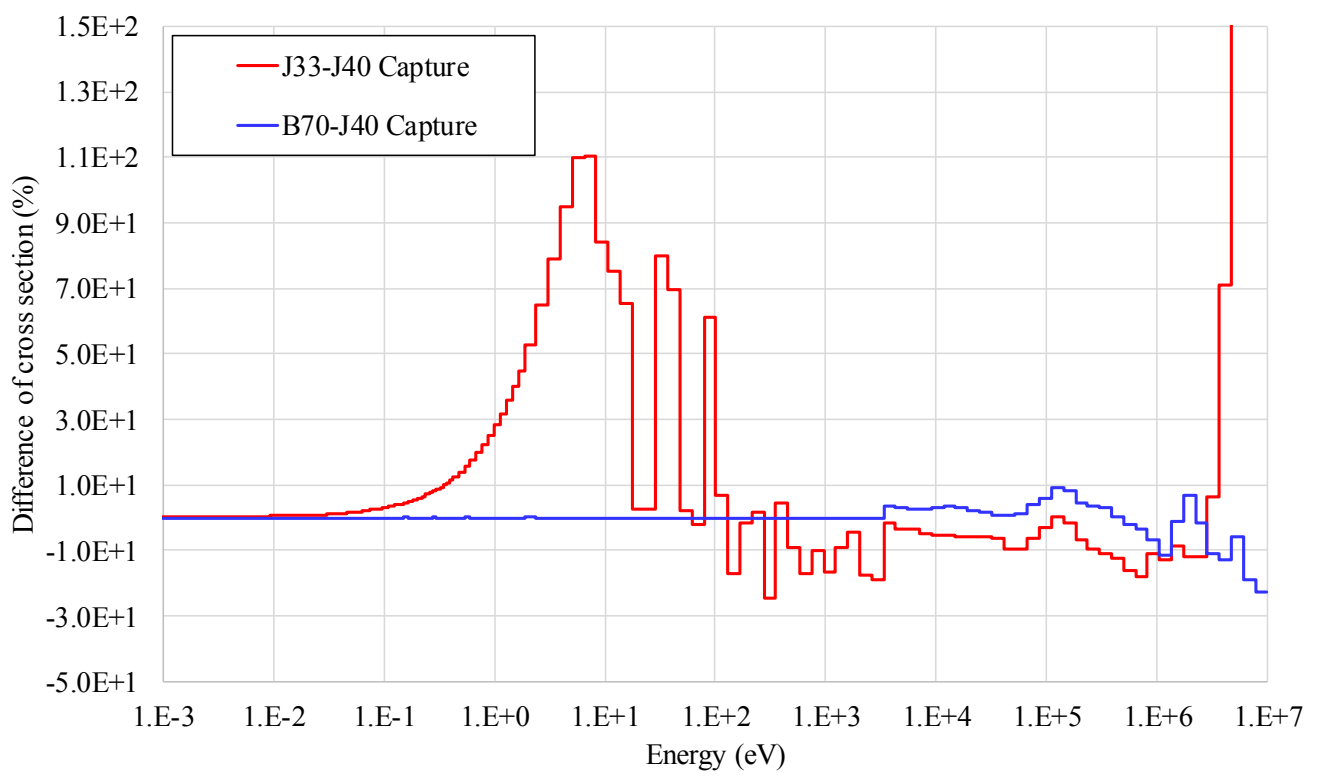

Figure 5. Differences of ${ }^{232} \mathrm{Th}$ capture cross section.

In order to quantitatively evaluate the contribution of the cross section difference for the $k_{\text {eff }}$, the sensitivity coefficient for effective multiplication factor defined by the following equation were calculated by sensitivity calculational code SAGEP [7].

$$
\begin{aligned}
& \mathrm{S}=\frac{\frac{d k_{\text {eff }}}{k_{\text {eff }}}}{\lambda \frac{d \sigma}{\sigma}}=\frac{\left\langle\psi \frac{d F}{d \sigma} \phi\right\rangle}{\langle\psi F \phi\rangle}-\frac{\left\langle\psi \frac{d A}{d \sigma} \phi\right\rangle}{\langle\psi A \phi\rangle}=\frac{\left\langle\psi \frac{d B}{d \sigma} \phi\right\rangle}{\lambda\langle\psi F \phi\rangle}, \\
& \lambda=\frac{1}{k_{\text {eff }}},
\end{aligned}
$$

Where, $\psi$ is adjoint neutron flux, neutron flux, $\mathrm{F}$ is operator for fission in neutron diffusion equation, and A is operator for leakage, absorption and scattering in neutron diffusion equation. By using the sensitivity coefficient and the cross section difference, it is possible to evaluate the energy depend contribution " $R$ " of the change in effective multiplication factor.

$$
\mathrm{R}=\mathrm{S} \times \frac{\Delta \sigma}{\sigma}
$$

Figure 6 shows the contribution of the $\mathrm{k}_{\text {eff }}$ difference due to the ${ }^{232} \mathrm{Th}$ capture cross section change when the nuclear data library is changed from JENDL-4.0 to JENDL-3.3 and ENDF / B-VII.0. In JENDL-3.3, it is observed the effect of increasing the capture cross section in the thermal energy region and decreasing the $\mathrm{k}_{\text {eff, }}$ and the effect of decreasing the cross section and increasing the $\mathrm{k}_{\text {eff }}$ at the energy region above the second resonance. Since these effects cancel each other, the difference of the $\mathrm{k}_{\text {eff }}$ between JENDL-4.0 and JENDL-3.3 is small. On the other hand, in ENDF/B-VII.0, the difference of the $\mathrm{k}_{\text {eff }}$ between JENDL-4.0 and ENDF / B-VII.0 becomes small due to the effect of increasing and decreasing the effective multiplication factor in the energy region above $3.3 \mathrm{keV}$. The difference of the $\mathrm{k}_{\mathrm{eff}}$ by the library change are almost the same, but the energy-wise contribution of cross section is different. 


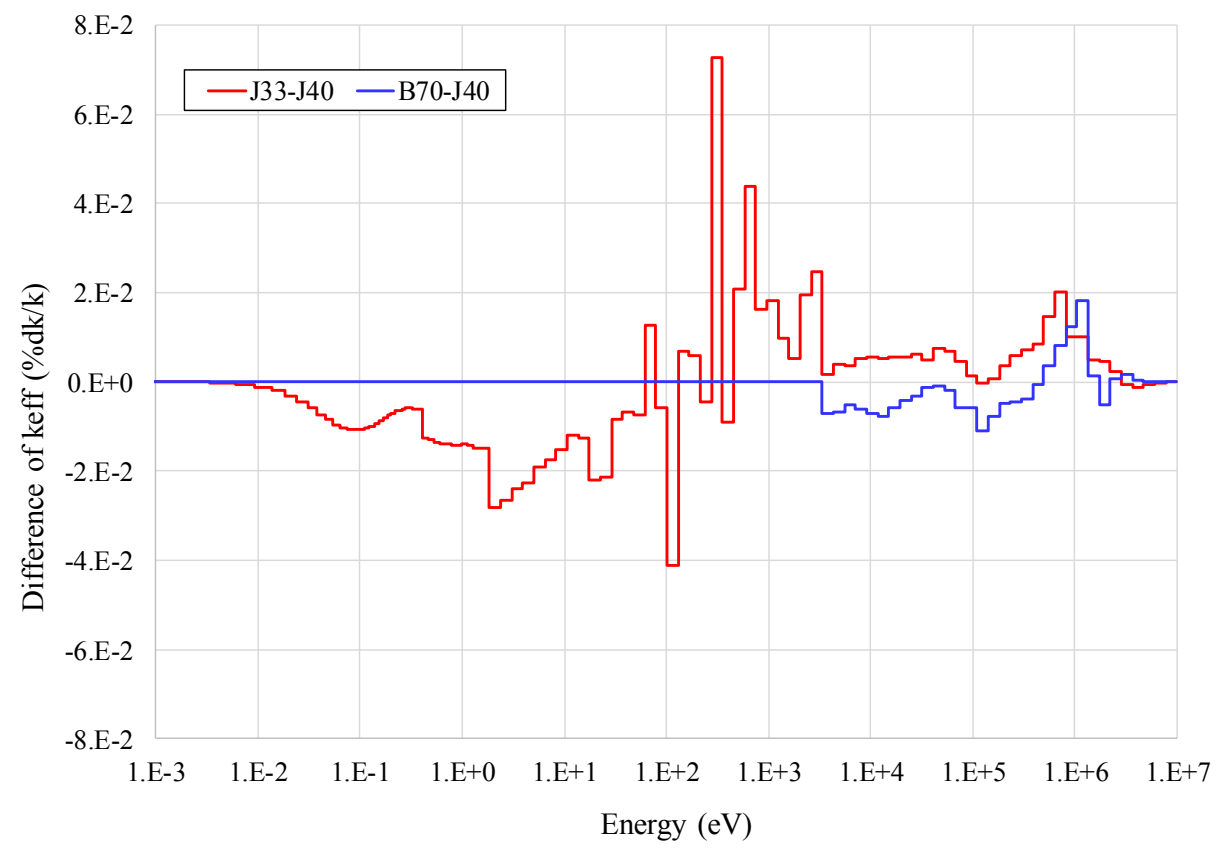

Figure 6. Energy-wise contributions of $k_{\text {eff }}$ difference.

\section{CONCLUSIONS}

In this study, from the viewpoint of expanding critical experimental data, the Th fuel loaded core with 70 of $\mathrm{H} /{ }^{235} \mathrm{U}$ and 12.7 of ${ }^{232} \mathrm{Th} /{ }^{235} \mathrm{U}$ ratio was newly constructed at KUCA. In order to obtain an effective multiplication factor of the core described in the previous section, excess reactivity measurement was performed 6 times by the reactor period method, respectively. As the results, the excess reactivity was $0.0865 \pm 0.0026(\% \mathrm{dk} / \mathrm{k})$ and the $\mathrm{k}_{\text {eff }}$ was $1.0009 \pm 0.0003$.

In the numerical analysis using continuous energy Monte-Carlo code MVP3.0, The respective calculations for the $\mathrm{k}_{\text {eff }}$ with JENDL-4.0, JENDL-3.3 and ENDF/B-VII.0 lead to $1.0056 \pm 0.0086$ (\%), $1.0048 \pm 0.0085(\%)$ and $1.0056 \pm 0.0086(\%)$. The $\mathrm{C} / \mathrm{E}$ values were 1.0047, 1.0039 and 1.0047, respectively.

In order to evaluate the impact of of the difference of ${ }^{232} \mathrm{Th}$ capture cross section among JENDL-4.0, JENDL-3.3 and ENDF/B-VII.0 to the $\mathrm{k}_{\mathrm{eff}}$, the sensitivity analysis were carried out. In the evaluation, only the ${ }^{232} \mathrm{Th}$ cross sections were taken from JENDL-4.0, JENDL-3.3 or ENDF/B-VII.0 but all other nuclides were done from JENDL-4.0. As the results, the differences of the $\mathrm{k}_{\text {eff }}$ due to ${ }^{232} \mathrm{Th}$ cross section change were obtained to be $0.180(\% \mathrm{dk} / \mathrm{k})$ of JENDL-3.3 and $0.162(\% \mathrm{dk} / \mathrm{k})$ of ENDF/B-VII.0. To quantitatively evaluate the contribution of the cross section difference to the $\mathrm{k}_{\text {eff }}$, the sensitivity coefficients for $k_{\text {eff }}$ were calculated by sensitivity calculational code SAGEP As the results, The difference of the $\mathrm{k}_{\text {eff }}$ between JENDL-4.0 and other libraries were almost the same, but the energy-wise contribution of the cross section change was different. 


\section{ACKNOWLEDGMENTS}

This study was supported by CHUBU Electric Power Co., Inc. This work is done in part under the visiting researcher's program of the research reactor institute, Kyoto University, in 2018.

\section{REFERENCES}

1. H. Unesaki, "Study on Verification of Nuclear Data for Nuclear Design of Next Generation Thermal Reactors," dissertation, Kyoto University, (2001).

2. Y. Nagaya, et. al., MVP/GMVP Version 3: General Purpose Monte Carlo Codes for Neutron and Photon Transport Calculations Based on Continuous Energy and Multigroup Methods," Japan Atomic Energy Agency, JAEA-Data/Code, 2016-018, (2017).

3. K. Shibata, et. al., "JENDL-4.0: A New Library for Nuclear Science and Engineering," J. Nucl. Sci. Technol. 48, pp. 1-30, (2011).

4. K. Shibata, et. al., "Japanese Evaluated Nuclear Data Library Version 3 Revision-3: JENDL-3.3," J. Nucl. Sci. Technol. 39, pp.1125-1136, (2002).

5. M. B. Chadwick, et. al, "ENDF/B-VII.0: Next Generation Evaluated Nuclear Data Library for Nuclear Science and Technology," Nucl. Data Sheets.107, pp.2931-3060, (2006).

6. H. Unesaki, T. Sano, et. al., "Critical Experiment of Thorium Loaded Core at KUCA (2) Comparison of criticality analysis for present and previous experiment," Abstract of AESJ 2019 annual meeting, Ibaraki University, Mar. 20 - 22, 1J12, (2019), (in Japanese).

7. A. Hara, T. Takeda, Y. Kikuchi, SAGEP: Two-Dimensional Sensitivity Analysis Code Based on Generalized Perturbation Theory, Japan Atomic Energy Resarch Institute, JAERI-M 84-027, (1984), (in Japanese). 